\title{
Clinical factors associated with the humoral immune response to influenza vaccination in chronic obstructive pulmonary disease
}

This article was published in the following Dove Press journal:

International Journal of COPD

24 December 2013

Number of times this article has been viewed

\author{
Karthik D Nath ${ }^{1,2}$ \\ Julie G Burel' \\ Viswanathan Shankar ${ }^{3}$ \\ Antonia L Pritchard' \\ Michelle Towers ${ }^{2}$ \\ David Looke ${ }^{1,2}$ \\ Janet M Davies' \\ John W Upham ${ }^{1,2}$ \\ 'The University of Queensland \\ (School of Medicine), Brisbane, \\ QLD, Australia; ${ }^{2}$ Princess Alexandra \\ Hospital, Brisbane, QLD, Australia; \\ ${ }^{3}$ Department of Epidemiology and \\ Population Health, Albert Einstein \\ College of Medicine, New York, \\ NY, USA
}

Background and objective: Individuals with chronic obstructive pulmonary disease (COPD) are at a high risk of developing significant complications from infection with the influenza virus. It is therefore vital to ensure that prophylaxis with the influenza vaccine is effective in COPD. The aim of this study was to assess the immunogenicity of the 2010 trivalent influenza vaccine in persons with COPD compared to healthy subjects without lung disease, and to examine clinical factors associated with the serological response to the vaccine.

Methods: In this observational study, 34 subjects (20 COPD, 14 healthy) received the 2010 influenza vaccine. Antibody titers at baseline and 28 days post-vaccination were measured using the hemagglutination inhibition assay (HAI) assay. Primary endpoints included seroconversion ( $\geq 4$-fold increase in antibody titers from baseline) and the fold increase in antibody titer after vaccination.

Results: Persons with COPD mounted a significantly lower humoral immune response to the influenza vaccine compared to healthy participants. Seroconversion occurred in $90 \%$ of healthy participants, but only in $43 \%$ of COPD patients $(P=0.036)$. Increasing age and previous influenza vaccination were associated with lower antibody responses. Antibody titers did not vary significantly with cigarette smoking, presence of other comorbid diseases, or COPD severity. Conclusion: The humoral immune response to the 2010 influenza vaccine was lower in persons with COPD compared to non-COPD controls. The antibody response also declined with increasing age and in those with a history of prior vaccination.

Keywords: COPD, human influenza, humoral immunity, influenza vaccines, vaccination

\section{Introduction}

Infection with influenza virus represents one of the most important and common comorbidities in patients hospitalized with chronic obstructive pulmonary disease (COPD). ${ }^{1}$ This association is unsurprising given the burden of viral respiratory tract infections in COPD exacerbations. ${ }^{2}$ Persons with COPD are a high priority for prophylaxis with the influenza vaccine. ${ }^{3}$ However, there is limited high level evidence on current influenza vaccine regimens in the COPD population. ${ }^{4}$

Universal recommendations for vaccination in the COPD population continue to be made despite the paucity of data available on influenza vaccine immunogenicity in this group. ${ }^{3}$ Guidelines for annual influenza vaccination in persons with COPD are largely based on observational studies. ${ }^{4}$ Though Nichol et al have reported that the influenza vaccine reduces mortality outcomes in those with chronic lung disease, ${ }^{5}$ there is now increasing recognition of selection bias whereby the under-vaccinated were more likely to be individuals with poor health, while those who are vaccinated may adopt
Correspondence: John W Upham School of Medicine, The University of Queensland, Level 5, TRI Building, Princess Alexandra Hospital, Woolloongabba, Brisbane,

QLD, Australia

Tel +6I 734437000

Fax +6I 731437779

Email j.upham@uq.edu.au

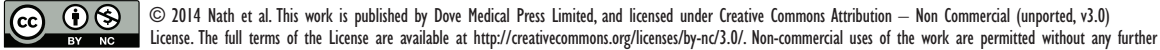
permission from Dove Medical Press Limited, provided the work is properly attributed. Permissions beyond the scope of the License are administered by Dove Medical Press Limited. Information on how to request permission may be found at: http://www.dovepress.com/permissions.php 
a range of other health-enhancing behaviors. ${ }^{6}$ Additionally, a recent randomized controlled trial from Thailand showing the effectiveness of influenza vaccination in COPD persons was conducted in a population that had not been previously vaccinated and used a dose of vaccine that was two-fold higher than that typically used in most parts of the world. ${ }^{7}$

The US Centers for Disease Control and Prevention appropriately summarized that the effectiveness of the influenza vaccine is primarily dependent on the age and immune competence of the vaccine recipient, and the similarities between influenza strains in the vaccine and those in circulation during any particular season. ${ }^{8}$ Because COPD patients often experience recurrent bacterial and viral infections, it has been proposed that they may be relatively immune-deficient compared to healthy persons, ${ }^{9}$ and as such may be less able to mount an effective immune response to vaccination. Immunogenicity to the influenza vaccine may also be affected by additional clinical factors such as prior vaccination status, current cigarette smoking, other comorbid chronic diseases, corticosteroid use, and COPD severity. ${ }^{7,10-13}$

This study was designed to assess the humoral immune response of the inactivated 2010 trivalent influenza vaccine in persons with COPD compared to non-COPD controls. Specific clinical factors considered to be associated with reduced vaccine immunogenicity, amongst all participants and within the COPD group, were also investigated.

\section{Methods}

\section{Study participants}

In this observational study, COPD participants were recruited from respiratory outpatient clinics of the Princess Alexandra Hospital, Brisbane, QLD, Australia. The nonCOPD controls were either spouses of patients or hospital staff. The PA Hospital Human Ethics Committee approved the study and written informed consent was obtained from all participants.

\section{Persons with COPD}

The key inclusion criterion was a known diagnosis of COPD using a post-bronchodilator forced expiratory volume in 1 second/forced vital capacity $\left(\mathrm{FEV}_{1} / \mathrm{FVC}\right) \leq 70 \%{ }^{14}$ The severity of COPD was determined using the GOLD criteria $^{14}$ and with DOSE scores. The DOSE index is a multidimensional staging system that includes dyspnea, airflow obstruction $\left(\mathrm{FEV}_{1}\right)$, smoking status, and exacerbations/year to measure COPD severity. ${ }^{15}$ All respiratory medications were permitted throughout the study. Those with COPD exacerbations or respiratory infections within 4 weeks before commencement of the study, invasive malignancy, acute febrile illness, and hypersensitivity to influenza vaccine or egg proteins were excluded.

\section{Healthy participants (controls)}

Healthy participants had no symptoms of lung disease and had normal spirometry with an $\mathrm{FEV}_{1}$ and FVC within the normal range. Key exclusion criteria were prior or current symptoms of lung disease, invasive malignancy, acute febrile illness, and hypersensitivity to influenza vaccine or egg proteins.

\section{Study design}

The prospective study consisted of two clinical visits (days 0 and 28). Eligible subjects received a single dose of the 2010 trivalent inactivated influenza vaccine $\left(\right.$ FluVax ${ }^{\circledR}$ 2010, CSL Biotherapies, Parkville, VIC, Australia) by deep subcutaneous injection on day 0. The Australian 2010 influenza vaccine contained $15 \mu \mathrm{g}$ of each of three influenza strains - the pandemic (H1N1) 2009 strain (A/California/7/2009), an H3N2 strain (A/Perth/16/2009), and a B strain (B/Brisbane/60/2008).

At baseline (day 0), information was collected via detailed questionnaire including participant demographics, other comorbid chronic diseases (diabetes mellitus, hypertension, cardiac illness, hypercholesterolemia, and/or stroke), medication use, previous influenza vaccination history, smoking status, and an assessment of COPD severity. Spirometry was performed in all subjects.

H1N1-vaccine specific antibodies were measured with the hemagglutination inhibition (HAI) assay performed on blood samples collected at day 0 (pre-vaccination) and day 28 (post-vaccination). Some participants had only a postvaccination blood sample. HAI testing was carried out at a regional reference laboratory (The Prince of Wales Hospital, Randwick, NSW, Australia).

The co-primary endpoints of this study were the percentage of participants achieving seroconversion (traditionally defined as $\geq 4$-fold increase in HAI antibody titers from baseline) and the fold increase in antibody level (the ratio of post-vaccination antibody titer to pre-vaccination antibody titer). The secondary outcome was absolute post-vaccination antibody titers.

\section{Statistical methods}

Descriptive statistics of titers were presented as median and range and other categorical covariates have been presented as frequencies and percentages. The association between COPD 
status and continuous covariates was analyzed using the Mann-Whitney $U$-test as data were not normally distributed. Those with categorical covariates were analyzed using the chi-square test. The within-group variability between pre- and post-vaccination antibody titers was analyzed using the Wilcoxon signed-rank test. The fold increase in titers between day 0 and day 28 was modeled using linear regression analysis. Since there were missing values on both outcome and covariates, we applied a multiple imputation procedure with the number of imputations being 20. The imputation model consisted of antibody titers at day 0, day 28, COPD status, previous vaccination status, age, and gender. The outcome variable fold increase in antibody titers was derived after imputing the values. The analysis model included covariates of COPD status, age, and prior vaccination status with fold increase as the outcome. All analyses were performed using SAS software (v9.3; SAS Institute Inc., Cary, NC, USA).

\section{Results}

Thirty-four (20 COPD, 14 healthy) study participants were enrolled from April to November, 2010. Patient demographics and clinical characteristics are presented in Table 1. COPD participants were significantly older with a mean age of 66 years

Table I Comparison of demographic and clinical characteristics between COPD and healthy participants

\begin{tabular}{|c|c|c|c|}
\hline & $\begin{array}{l}\text { COPD } \\
(\mathrm{N}=20) \\
\mathrm{N}(\%)\end{array}$ & $\begin{array}{l}\text { Non-COPD } \\
(\mathrm{N}=14) \\
\mathrm{N}(\%)\end{array}$ & $P$-value \\
\hline \multicolumn{4}{|l|}{ Pre-titer } \\
\hline Median (range) & $320(80,1,280)^{\wedge}$ & $60(20,640)^{\wedge \wedge}$ & $0.033^{\#}$ \\
\hline \multicolumn{4}{|l|}{ Post-titer } \\
\hline Median (range) & $640(160,1,280)$ & $1,280(160,1,280)$ & $0.024^{\#}$ \\
\hline \multicolumn{4}{|l|}{ Age (years) } \\
\hline Mean (SD) & $66.2(11.0)$ & $54.3(14.5)$ & 0.012 \\
\hline \multicolumn{4}{|l|}{ Sex } \\
\hline Male & $13(65)$ & $8(57.1)$ & 0.643 \\
\hline $\begin{array}{l}\text { Previous vaccination } \\
\text { status }\end{array}$ & $18(90)$ & II (78.6) & 0.354 \\
\hline \multicolumn{4}{|c|}{ Other comorbid diseases } \\
\hline Yes* & $7(35)$ & $2(14.3)$ & 0.18 \\
\hline \multicolumn{4}{|l|}{ Current smokers } \\
\hline Yes & $9(45)$ & 0 & $0.004^{\$}$ \\
\hline $\mathrm{ICS}^{\dagger}$ use & $14(70)$ & - & NA \\
\hline \multicolumn{4}{|l|}{$\mathrm{FEV}_{1}$} \\
\hline$\geq 80 \%$ & I (5) & & \\
\hline $50 \% \leq \mathrm{FEV}_{1}<80 \%$ & $9(45)$ & & \\
\hline $30 \% \leq \mathrm{FEV},<50 \%$ & $3(15)$ & & \\
\hline$<30 \%$ & $7(35)$ & & \\
\hline
\end{tabular}

Notes: ${ }^{\wedge}(\mathrm{N}=7) ;{ }^{\wedge \wedge}(\mathrm{N}=10) ;{ }^{*}$ Mann-Whitney U-Test; *diabetes mellitus, hypertension, cardiac illness, hypercholesterolemia, and/or stroke; 'Fisher's exact test.

Abbreviations: FEV, forced expiratory volume in I second; ICS, inhaled corticosteroids; SD, standard deviation; COPD, chronic obstructive pulmonary disease; $\mathrm{N}$, number of subjects; NA, not applicable. compared to 54 years in the healthy group $(P=0.012)$. None of the healthy participants were current cigarette smokers whereas nine (45\%) COPD persons were current smokers $(P=0.004)$. No significant difference was observed in gender and previous vaccination status amongst the two study groups. Of the COPD participants, 14 (70\%) were on inhaled corticosteroids (ICS), while one was also taking oral steroid medication. COPD patients had varying degrees of airflow obstruction: seven $(35 \%)$ had an $\mathrm{FEV}_{1}<30 \%$, three $(15 \%)$ had an $\mathrm{FEV}_{1}$ between $30 \%-50 \%$, nine (45\%) had an $\mathrm{FEV}_{1}$ between $50 \%-70 \%$, and one patient ( $5 \%$ ) had $\mathrm{FEV}_{1} \geq 80 \%$. The mean DOSE score in the COPD group was 2.8 (SD: 1.7). Of the 34 subjects, 17 (7 COPD, 10 healthy) had pre- (day 0) and post-vaccination (day 28) blood samples collected for antibody measurement. The remaining 17 participants (13 COPD, 4 healthy) had a post-vaccination blood measurement only.

Pre-vaccination HAI antibody titers were significantly higher in COPD patients (median $=320$ ) than in healthy participants (median $=60 ; P=0.033$ ). In contrast, postvaccination antibody titers were significantly lower in COPD patients (median: 640) than in healthy participants (median: 1,$280 ; P=0.024$ ).

Influenza vaccination produced a significant increase in antibody titers between day 0 and day 28 within the healthy group (mean increase: 1,086; SD: 245; $P=0.002$ ). The mean increase in antibody titers post-vaccination in the COPD group was 514 (SD: 510) and did not reach statistical significance ( $P=0.063$; Figure 1$)$.

After receiving the influenza vaccine, $90 \%$ of healthy participants seroconverted (traditionally defined as a fourfold or greater increase in antibody titer), whereas seroconversion occurred in only $43 \%$ of COPD patients $(P=0.036$; Figure 2). The healthy participants also had significantly

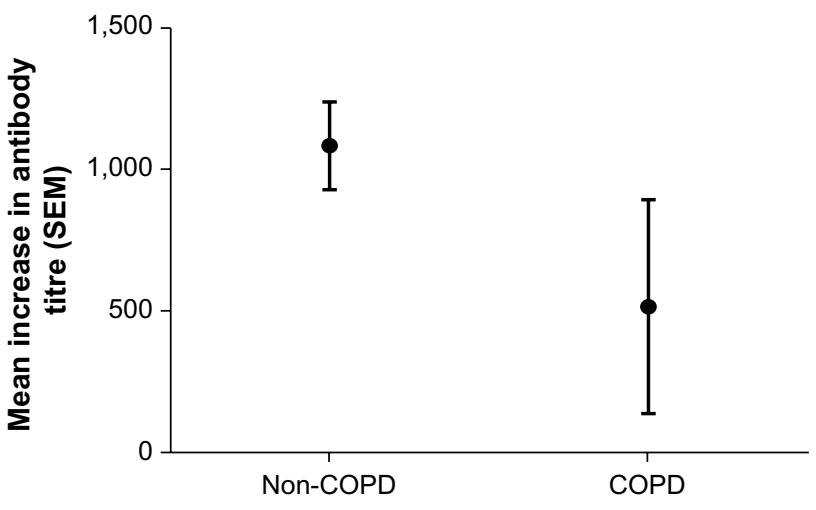

Figure I Mean increase in antibody titer (SEM) between day 0 (baseline) and day 28 post-vaccination within each study group.

Notes: Non-COPD, $P=0.002$; COPD, $P=0.063$ - Wilcoxon signed-rank test.

Abbreviation: SEM, standard error mean; COPD, chronic obstructive pulmonary disease. 


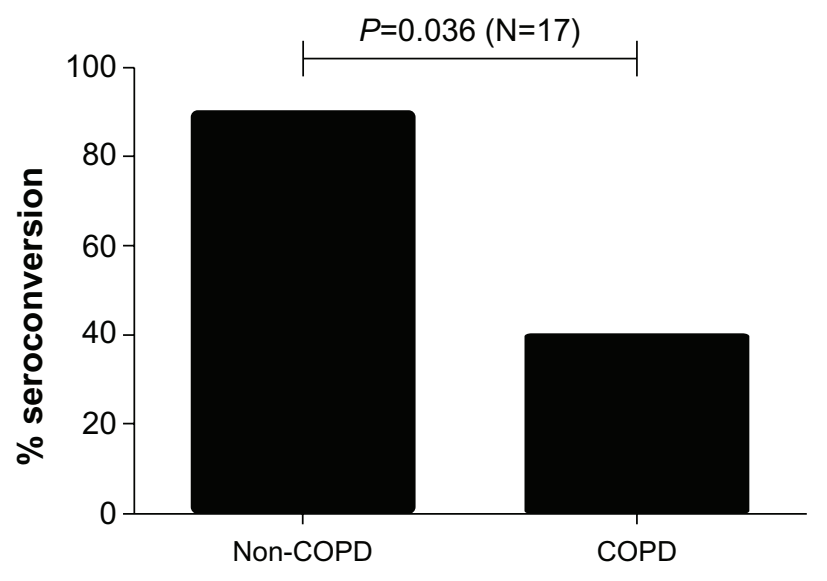

Figure 2 Comparison of the percentage of subjects that seroconverted ( $\geq 4$-fold increase in antibody titers from baseline) between the COPD and non-COPD groups. Note: $P=0.036$ - chi-square test.

Abbreviations: $\mathrm{N}$, number of subjects; COPD, chronic obstructive pulmonary disease.

higher fold increases in antibody titer than the COPD patients $(P=0.002)$. Seroprotection, which is traditionally defined as an HAI titer $\geq 40$, was observed in all healthy participants and all COPD patients after vaccination.

Linear regression modeling with antibody titers as the outcome variable showed that COPD was significantly and independently associated with a reduced antibody response to the vaccine, even after adjusting for age and previous vaccination status (Table 2). The analysis showed an estimated average 10.3-fold lower change in antibody titers in those with COPD compared to the healthy group and this was statistically significant $(P=0.019)$. A trend towards association was observed between age of study participants and antibody titers; for each 1 -year increase in age, the fold change in antibody titer was lower by 0.3 units $(P=0.078$ ). The fold change in antibody titers after vaccination tended to be lower in those with previous H1N1 vaccination though this was not statistically significant $(P=0.345)$. No association was observed between vaccine response and being a current cigarette smoker, presence of other comorbid chronic disease, ICS use, or COPD severity.

\section{Discussion}

COPD represents an important and increasing burden throughout the world with influenza infection playing a

Table 2 Multiple linear regression analysis of factors associated with antibody titers

\begin{tabular}{lll}
\hline Variables & $\beta$ (SE) & $P$-value \\
\hline COPD status & $-10.28(4.37)$ & 0.019 \\
Age & $-0.26(0.15)$ & 0.078 \\
Previous vaccination status & $-3.53(3.73)$ & 0.345 \\
\hline
\end{tabular}

Note: $\beta$ coefficient, standard error, and $P$-value from a multiple imputation linear regression model are shown.

Abbreviation: SE, standard error; COPD, chronic obstructive pulmonary disease. significant role in COPD exacerbations. ${ }^{7}$ There is limited data on influenza vaccine immunogenicity in the COPD population. ${ }^{4}$ We therefore sought to investigate the humoral immune response to the influenza vaccine in persons with COPD and found it to be significantly lower compared to their non-COPD counterparts. The HAI antibody response was also seen to be reduced in those of older age.

The influenza virus envelope protein hemagglutinin (HA) binds to erythrocytes. Inhibition of hemagglutination forms the basis of the HAI assay in determining levels of antibody. It has been well-documented that anti-HA antibody titers, induced post-vaccination by host antigen-specific B cells and measured with the HAI assay, correlates positively with clinical protection against influenza. ${ }^{16}$

Though COPD depresses immunity, ${ }^{9,17}$ there are few studies comparing antibody responses to the influenza vaccine in persons with COPD to healthy volunteers. A Cochrane review of influenza vaccine effectiveness in those with COPD did not evaluate antibody response as an outcome of interest. ${ }^{4}$ We found that persons with COPD mounted a lower serological immune response to the influenza vaccine compared to those without COPD. After receiving the vaccine, $43 \%$ of COPD individuals seroconverted in contrast to $90 \%$ of healthy volunteers. These findings provide the impetus for more research into improving influenza vaccination strategies in persons with COPD.

A recent systematic review found that no firm conclusions could be drawn on the effectiveness of the influenza vaccine in the elderly due to the poor quality of the currently available evidence. ${ }^{11}$ Like those with COPD, the elderly are vulnerable to influenza infection, often with significant morbidity and mortality risks. Our study has shown that antibody responses to the influenza vaccine decline with increasing age, in agreement with previous reports in healthy cohorts. ${ }^{18-20}$

We found that antibody responses to vaccination were lower in those who received the influenza vaccine in the past. As baseline antibody titers were higher in persons with a prior vaccination history, our data suggests that reductions in seroconversion rates may be secondary to the high baseline titers that have already reached a plateau in these persons - somewhat of a mathematical artefact. ${ }^{21}$ Another possible explanation is that of 'original antigenic sin', whereby immunological memory from prior vaccinations traps the recipient immune system from mounting an effective response to subsequent vaccine strains of the influenza virus. $^{22}$ Prior vaccination continued to offer protection levels as seen with high baseline antibody titers, indicating that these patients need to continue to be vaccinated annually to 
maintain protection titers and develop immunity to antigenically different influenza strains.

Current cigarette smoking status, ICS use, and COPD severity were not seen to be predictors of a reduced serological immune response, as determined by the primary endpoints. However, absolute post-vaccination titers were significantly lower in persons using ICS. While this might be explained by systemic absorption of ICS, it is also feasible that use of ICS may be simply a marker of COPD severity. Further studies are required to determine whether smoking status, COPD severity, and/or ICS use independently affect influenza vaccine immunogenicity.

Larger studies are needed to validate our observations in the COPD population. It may then be necessary to adapt current influenza vaccine formulations to the varied immune responses of recipients. The observation that the efficacy of vaccinations is reduced in the elderly has already prompted investigation of altered vaccine formulations, either with an increased dose or the addition of adjuvants. ${ }^{23,24}$ Busse and colleagues found that administration of a $30 \mu \mathrm{g}$ dose of the monovalent $2009 \mathrm{H} 1 \mathrm{~N} 1$ influenza vaccine provided an increased immune response to vaccination in elderly asthmatics with severe disease compared to a standard $15 \mu \mathrm{g}$ dose. ${ }^{23}$ Studies have also shown that the addition of the adjuvant MF-59 to influenza vaccine formulations confers superior protection than a conventional subunit vaccine in adults affected by chronic diseases. ${ }^{24}$

There are limitations to this study. Serological responses to only one of three viral strains present in the 2010 trivalent influenza vaccine were measured. It is possible that antibody responses vary against the three viral strains of the vaccine. Additionally, the small sample size limits the statistical power of our observations. Participant recruitment was restricted by the short duration from when the influenza vaccine became available to vaccine administration prior to the anticipated winter outbreak of influenza. It would have been ideal for all participants to have both pre- and post-vaccination antibody measurements, though the multiple imputation procedure corrected for the missing pre-vaccination titers. It is acknowledged that the COPD patients were older than the healthy participants. However, the multiple regression analysis indicates that COPD was significantly and independently associated with a reduced antibody response to the vaccine after correcting for potential confounding factors including age.

In conclusion, our results demonstrate that persons with COPD mount a lower humoral immune response to the 2010 influenza vaccine compared to healthy volunteers.
This complements our recent study in which we used the same dataset to investigate cellular and molecular mechanisms to influenza vaccination, finding that serum IL-21, B-cell proliferation, and T-cell IFN- $\gamma$ production were lower in COPD patients. ${ }^{25}$ Increasing age and prior influenza vaccination were associated with a significant reduction in vaccine immunogenicity. While larger studies are needed to confirm our findings, our study provides the impetus for further research aimed at improving vaccine effectiveness in COPD.

\section{Disclosure}

The authors report no conflicts of interest in this work.

\section{References}

1. Rothbarth PH, Kempen BM, Sprenger MJ. Sense and nonsense of influenza vaccination in asthma and chronic obstructive pulmonary disease. Am J Respir Crit Care Med. 1995;151:1682-1685.

2. De Serres G, Lampron N, La Forge J, et al. Importance of viral and bacterial infections in chronic obstructive pulmonary disease exacerbations. J Clin Virol. 2009;46:129-133.

3. Pesek R, Lockey R. Vaccination of adults with asthma and COPD. Allergy. 2011;66:25-31.

4. Poole PJ, Chacko E, Wood-Baker RW, Cates CJ. Influenza vaccine for patients with chronic obstructive pulmonary disease. Cochrane Database Syst Rev. 2006;(1):CD002733.

5. Nichol KL, Baken L, Nelson A. Relation between influenza vaccination and outpatient visits, hospitalization, and mortality in elderly persons with chronic lung disease. Ann Intern Med. 1999;130:397-403.

6. Goodwin K, Viboud C, Simonsen L. Antibody response to influenza vaccination in the elderly: a quantitative review. Vaccine. 2006;24: 1159-1169.

7. Wongsurakiat P, Maranetra KN, Wasi C, Kositanont U, Dejsomritrutai W, Charoenratanakul S. Acute respiratory illness in patients with COPD and the effectiveness of influenza vaccination: a randomized controlled study. Chest. 2004;125:2011-2020.

8. Harper SA, Fukuda K, Uyeki TM, Cox NJ, Bridges CB; Centers for Disease Control and Prevention (CDC) Advisory Committee on Immunization Practices (ACIP). Prevention and control of influenza: recommendations of the Advisory Committee on Immunization Practices (ACIP). MMWR Recomm Rep. 2004;53:1-40.

9. Yerkovich ST, Hales BJ, Carroll ML, et al. Reduced rhinovirusspecific antibodies are associated with acute exacerbations of chronic obstructive pulmonary disease requiring hospitalisation. BMC Pulm Med. 2012;12:37.

10. Cruijff M, Thijs C, Govaert T, Aretz K, Dinant GJ, Knottnerus A. The effect of smoking on influenza, influenza vaccination efficacy and on the antibody response to influenza vaccination. Vaccine. 1999;17: 426-432.

11. Jefferson T, Di Pietrantonj C, Al-Ansary LA, Ferroni E, Thorning S, Thomas RE. Vaccines for preventing influenza in the elderly. Cochrane Database Syst Rev. 2010;(2):CD004876.

12. Gross PA, Quinnan GV Jr, Weksler ME, Setia U, Douglas RG Jr. Relation of chronic disease and immune response to influenza vaccine in the elderly. Vaccine. 1989;7:303-308.

13. Hanania NA, Sockrider M, Castro M, et al; American Lung Association Asthma Clinical Research Centers. Immune response to influenza vaccination in children and adults with asthma: effect of corticosteroid therapy. J Allergy Clin Immunol. 2004;113:717-724.

14. Gomez FP, Rodriguez-Roisin R. Global Initiative for Chronic Obstructive Lung Disease (GOLD) guidelines for chronic obstructive pulmonary disease. Curr Opin Pulm Med. 2002;8:81-86. 
15. Jones RC, Donaldson GC, Chavannes NH, et al. Derivation and validation of a composite index of severity in chronic obstructive pulmonary disease: the DOSE Index. Am J Respir Crit Care Med. 2009;180:1189-1195.

16. Halliley JL, Kyu S, Kobie JJ, et al. Peak frequencies of circulating human influenza-specific antibody secreting cells correlate with serum antibody response after immunization. Vaccine. 2010;28:3582-3587.

17. Agusti A, Soriano JB. COPD as a systemic disease. COPD. 2008;5: 133-138.

18. D’Alessio DJ, Cox PM Jr, Dick EC. Failure of inactivated influenza vaccine to protect an aged population. JAMA. 1969;210:485-489.

19. Howells CH, Vesselinova-Jenkins CK, Evans AD, James J. Influenza vaccination and mortality from bronchopneumonia in the elderly. Lancet. 1975;1:381-383.

20. Phair J, Kauffman CA, Bjornson A, Adams L, Linnemann C Jr. Failure to respond to influenza vaccine in the aged: correlation with B-cell number and function. J Lab Clin Med. 1978;92:822-828.

21. Beyer WE, Palache AM, Sprenger MJ, et al. Effects of repeated annual influenza vaccination on vaccine sero-response in young and elderly adults. Vaccine. 1996;14:1331-1339.
22. Webster RG, Kasel JA, Couch RB, Laver WG. Influenza virus subunit vaccines. II. Immunogenicity and original antigenic sin in humans. J Infect Dis. 1976;134:48-58.

23. Busse WW, Peters SP, Fenton MJ, et al. Vaccination of patients with mild and severe asthma with a 2009 pandemic H1N1 influenza virus vaccine. J Allergy Clin Immunol. 2011;127:130-137, 137. e1-e3.

24. Baldo V, Baldovin T, Floreani A, Carraro AM, Trivello R; Family Medicine Group of Pianiga. MF59-adjuvanted influenza vaccine confers superior immunogenicity in adult subjects (18-60 years of age) with chronic diseases who are at risk of post-influenza complications. Vaccine. 2007;25:3955-3961.

25. Burel JG, Nath K, Pritchard AL, et al. Impaired immune response to influenza vaccination in chronic obstructive pulmonary disease. J Vaccines Vaccin. 201; http://dx.doi.org/10.4172/2157-7560.S4-001. Accessed May 1, 2013.

\section{Publish your work in this journal}

The International Journal of COPD is an international, peer-reviewed journal of therapeutics and pharmacology focusing on concise rapid reporting of clinical studies and reviews in COPD. Special focus is given to the pathophysiological processes underlying the disease, intervention programs, patient focused education, and self management protocols.

\section{Dovepress}

This journal is indexed on PubMed Central, MedLine and CAS. The manuscript management system is completely online and includes a very quick and fair peer-review system, which is all easy to use. Visit http://www.dovepress.com/testimonials.php to read real quotes from published authors. 